\title{
Implications of the Dunbar Number in Collective Intelligence based on Social Network Services
}

\author{
Tae-Won Kim \\ Ph.D. candidate, Graduate School of Chungbuk National University \\ \#52 Naesudong-ro Heungduk-gu, Cheongju, Chungbuk, 361-763, South Korea \\ e-mail: joonari@naver.com, Tel: +82-43-271-1894 \\ Jae-Rim Jung \\ Ph.D. candidate, Graduate School of Chungbuk National University \\ \#52 Naesudong-ro Heungduk-gu, Cheongju, Chungbuk, 361-763, South Korea \\ e-mail: jeremy80@naver.com, Tel: +82-43-271-1894 \\ Sang-Wook Kim \\ Professor, Department of MIS, Chungbuk National University \\ \#52 Naesudong-ro Heungduk-gu, Cheongju, Chungbuk, 361-763, South Korea \\ e-mail: sierra@cbnu.ac.kr, Tel: +82-43-261-2357
}

\begin{abstract}
This study establishes a simple Causal Map in order to understand the structure of Social Network Services(SNS). From this, the paper proposes a simulation with drawing Simulation Model and it examines whether a Dunbar Number is available for the emergence of collective intelligence based on an on-line network. Through my analytical research, it turn out both Closed SNS and Open SNS have their own Dunbar Number. However, it appear that Open SNS can expand infinitely as it has a unique property namely weak tie. This implies that it is significant for a system or policy to be developed in order to overcome the problem of Dunbar Number, whereas restricting the extension of a network by considering the Dunbar Number would have a negative impact on emergence of collective intelligence.
\end{abstract}

Keywords: Dunbar Number, Collective Intelligence, Social Network Services, System Dynamics

\section{INTRODUCTION}

It is perhaps a common sense that collective intelligence is embodied more effectively as more people participate in the process. It is, however, also questionable whether a group of people depends upon intelligence. There are often people who are outstanding individually, but show an extremely different performance collectively. That is, collective intelligence and collective stupidity are another matter regardless how smart each individual is. No matter how outstanding experts individually are, the group consisting of those experts may show a poor performance [1], [2], [4] and in contrast, a group of stupid people may yield a wise result [3], [7].

According to Robin Dunbar, the most sociable and outgoing

\footnotetext{
* Corresponding author,Email: joonari@naver.com Manuscript received Apr. 12, 2012; revised Sep 17, 2012; accepted Sep 20, 2012
}

people can maintain a close interpersonal relation at most with 150 individuals [8]. People tend to form a community in order to obtain a rightly mutual understanding and cooperation with others, but the stable structure of mutual understanding and cooperative relation is likely to be broken down as the number of individuals in a group exceeds 150 , so that it is needed to develop effective rules, laws, and policies in order to maintain the group in which more than 150 people participate.

Previously numerous studies on Dunbar Number have supported that the Number applies to all the communities concerned. However, these studies do not provide us with a clear evidence that explains why the more users in a representative collective intelligence Wikipedia mean the more accurate information or why Twitter maintains collective intelligence consistently even in case where more than 150 followers. Since prior studies attempted to analyze the relation focused upon specific services rather than the structural characteristics and behavioral patterns of Social Networking 
Services(SNS), these have a limitation that it cannot consider the feedback resulting from the mutual impacts between relevant internal factors and structural characteristics of such services.

Thus the study draws a causal map, designs a simulation model based upon the map, and implements the simulation, which ultimately explores whether the theory of Dunbar Number is valid in creating collective intelligence based on online network, in order to examine the structure of SNS.

\section{SISTEM DYNAMICS}

The existing SNS-related researches weren't able to consider feedback created by mutual effects among inherent related factors and service's structural characteristics, since they mostly focused on fragmentary, particular services to analyze relations. This ignores the effect of feedback according to the flow of time, although it is able to predict an exact value, when inferring relations at a particular point of time. For SNS which consider interaction as the core, particularly, this concern becomes more serious. This study is intended to dynamically show a feedback phenomenon from each element according to the flow of time, using the system dynamics methodology in order to remedy this shortcoming.

The system dynamics methodology is more interested in the tendency of dynamic changes(stable/unstable tendency, periodic up-and-down wave, growth/decline pattern and maintenance of an equilibrium state) depending on the flow of time of variable which is the subject of interest, rather than measuring an exact model parameter value or yielding variable's estimate, because it basically gives a focus on how a particular variable changes dynamically, as time passes by [5].

\section{SIMULATION MODEL}

\subsection{Causal Loop Diagram of Closed SNS}

SNS is categorized into several types according to usage motive and function, but profile-based Facebook, Myspace in the United States and Cyworld, Kakaostory in Korea belong to closed SNS. Such closed form of networking service is used to build and maintain a friendly relation with people. In these services, it functions in a way that a person asks others to agree to the connection with each other, and then the person asked determining whether to connect or not. The Causal Map in Figure 1 illustrates the structure of closed SNS.

$\mathrm{R} 1$ loop is a self-reinforcing loop which helps to form a network of friends with usability, increases the number of human networks(the number of friends) who are strongly connected, and expands a relationship so the network formed can make another friend. This is like a growth engine in the system. However, the large number of strongly connected human networks increases complexity and reduces capacity of management, because human is limited in a cognitive power.

This corresponds to Dunbar's Number that Robin Dunbar insists. Because Dunbar's Number makes it impossible to form relationships above a certain level, the number of strongly connected human networks would remain at a certain standard.
This self-reinforcing B1 loop demonstrates that system is the archetype of typical 'limit of growth' which stops growing at a limited level or declines, not infinitely growing.

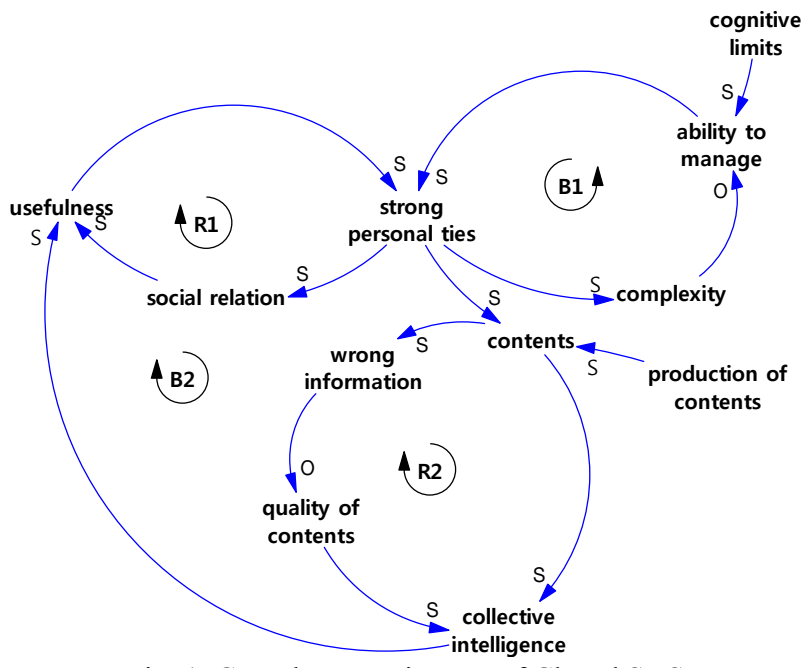

Fig. 1. Causal Loop Diagram of Closed SNS

The increase of strongly connected human networks leads to mass-production of contents. Also, there would be the contents produced by yourself here, too. Therefore, 'Production of Contents' was added as an exogenous variable. Collective intelligence may be improved, as there are more contents produced by yourself or others, but on the other hand it would hinder collective intelligence in some cases, by increasing wrong information and downgrading the quality of contents, relatively.

In particular, it is more serious in the way that collective thinking to hamper collective intelligence appears better, when there are strong ties that are already acquainted with each other. Thus, the variables, 'Wrong Information' and 'the Quality of Contents' were added to show positive and negative effects of contents on collective intelligence, at the same time. In other words, the increase of collective intelligence improves cognitive usability and again, this usability enlarges strong ties and increases contents and another R2 loop and more contents to enhance collective intelligence are produce, but wrong information created by contents leads to the B2 loop which declines collective intelligence.

\subsection{Causal Loop Diagram of Open SNS}

There are Open SNS as well as Closed SNS. Open SNS has an attribute that it maintains a number of links with weak connectivity and thus a broader intellectual base compared to Closed SNS that has a strong, complete, and closed structure. In addition, Open SNS is advantageous in that it secures the diversity and independence that prevents participants from falling into a group think, and in which self purification process is taking place in case of the inflow of misinformation through a weakly connected link, which leads to more reliable information. In this study, the author examines Twitter as a typical example of Open SNS. Twitter has a unique feature that it maintains a weak tie by following of one party compared to existing community or network adopting a bilateral tie. Figure 
2 illustrates the Causal Map that explains about the structure of Twitter.

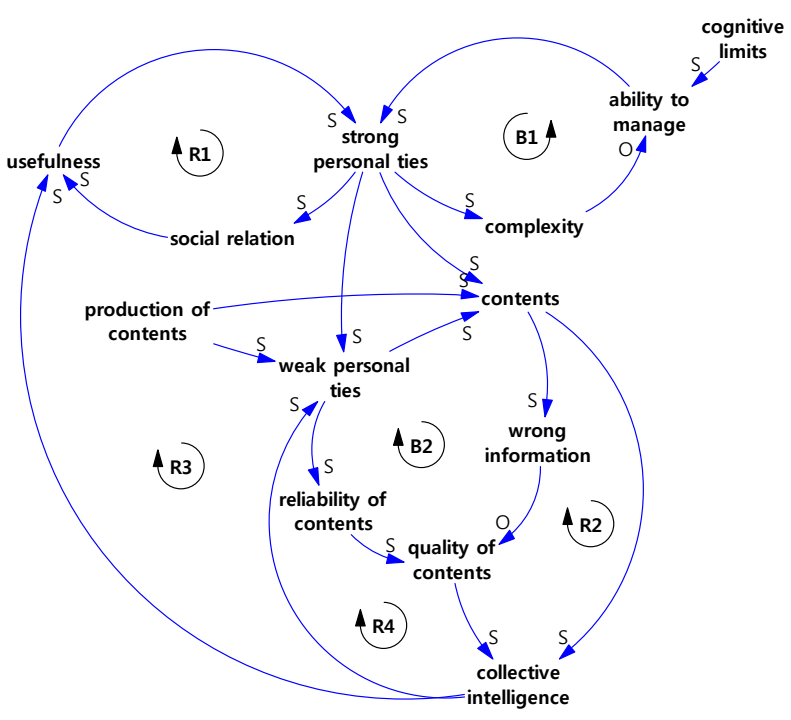

Fig. 2. Causal Loop Diagram of Open SNS

R1 loop and B1 loop are explained in Figure 1. Twitter has not only strong ties, but also weak ties. But here, the human networks that individuals have followed, are regarded as strong ties, not the cases to follow each other. As explained previously, Dunbar's Number is associated with cognitive levels of individuals, because followers on Twitter are whom you are aware of and manage. On the contrary, weak ties are the followers who don't have big influences on your cognitive ability. They increase according to the number of strongly connected human networks, or the contents created by themselves or collective intelligence.

As strong ties do, weak ties produce contents in large quantities, and positively affects collective intelligence(R2 loop) or negatively affects it(B2 loop), by lowering the quality of contents with wrong information. However, non-redundant information is distributed more diversely and it brings about spread, mix, sharing and exchange of new information in a state of weak ties, in contrast to strong ties that are closely connected. Moreover, it maintains independence, because there isn't much mutual interference and there are narrow and varied ties, due to the division of power and members under the weak ties with special knowledge purify wrong information by themselves through adjustment.

This process upgrades the reliability and quality of contents. The improved quality of contents means that collective intelligence of the environmental composition gets better(R4 loop). The strengthened collective intelligence motivates weak ties and strong ties(R3 loop), that is, Twitter to increase following and followers(R4 loop).

\subsection{Stock Flow Diagram}

The author designed the Stock Flow Diagram based upon the Causal Map as in Figure 3 and Figure 4.

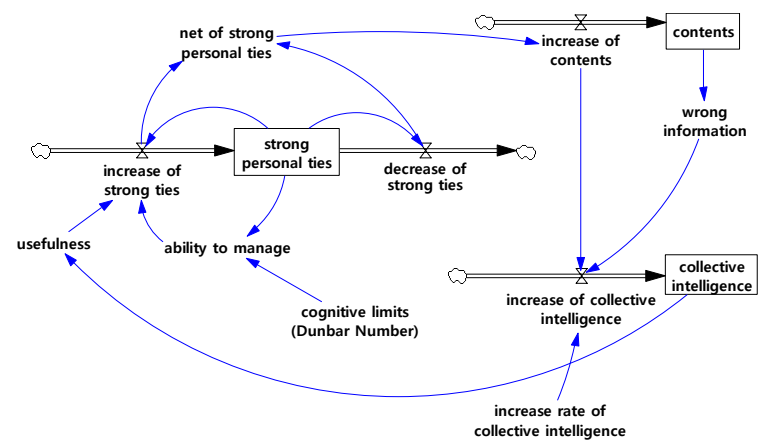

Fig. 3. Stock Flow Diagram of Open SNS

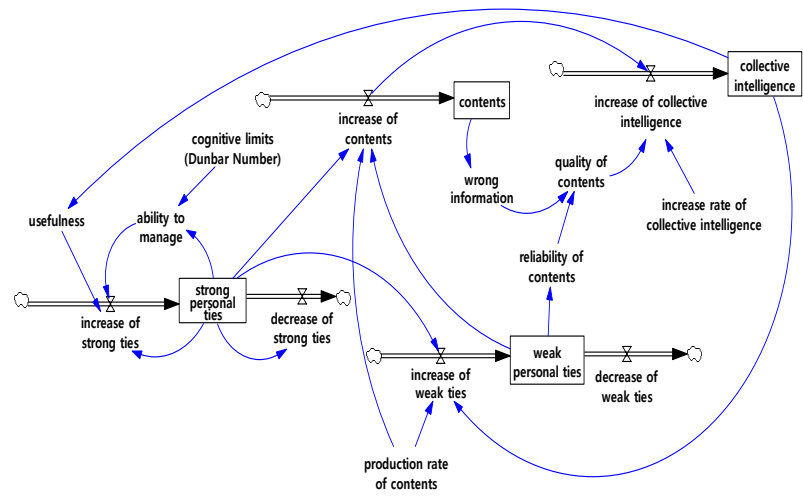

Fig. 4. Stock Flow Diagram of Open SNS

\section{THE RESULT OF SIMULATION}

\subsection{The Result of Simulation of Closed SNS}

The simulation on Closed SNS illustrates the dynamic structure where a SNS user begins to make the first strong tie and the corresponding changes in the number of strong personal ties and the increasing contents, which functions to activate the collective intelligence. In this simulation, the author intentionally adjusts the values for the factors that have an impact on the structure, and examine whether Dunbar Number applies to Closed SNS and how the intentional increase in the number of strong personal ties affects the contents of collective intelligence based upon the simulation result.

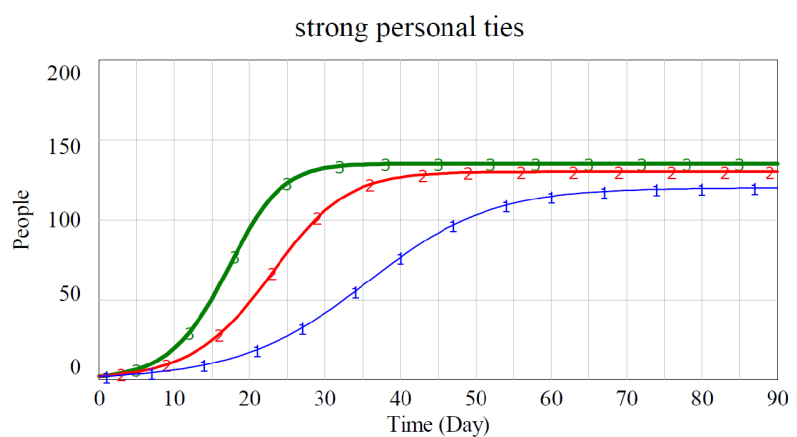

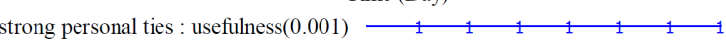
strong personal ties : usefulness $(0.0015)$ strong personal ties : usefulness $(0.002)$

Fig. 5. The Changes in the Number of Strong Personal Ties (Closed SNS) 


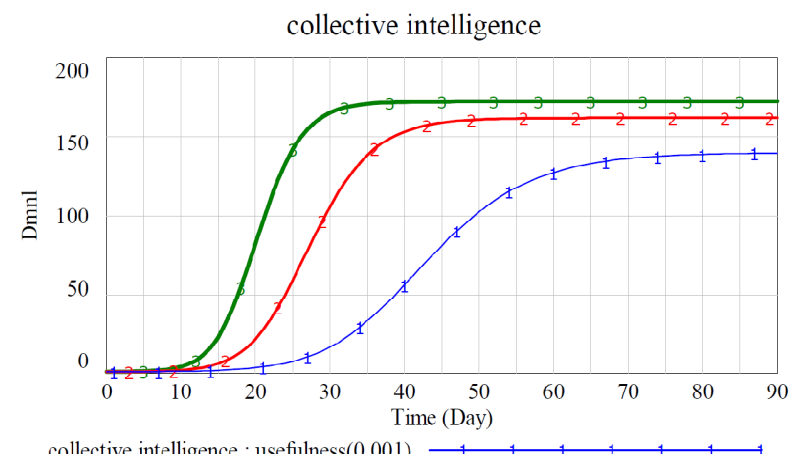

collective intelligence : usefulness $(0.001) \quad \begin{array}{lllllll}1 & 1 & 1 & 1 & 1 & 1\end{array}$ collective intelligence : usefulness $(0.0015)$ collective intelligence : usefulness $(0.002)$

Fig. 6. The Changes in Collective Intelligence (Closed SNS)

The author modifies the usefulness value in order to identify whether Dunbar Number applies to Closed SNS as Figure 5. The simulation reveals that the number of strong personal ties does not exceed a certain level imposed by Dunbar Number even in case where there is a change in usefulness. The changes in usefulness value have no impact on the structure of BOT(Behavior of time). The only difference was that how rapidly it converges into a goal or threshold. This shows that Dunbar Number still applies to Closed SNS as well. That is, collective intelligence is not increased beyond a certain level due to Dunbar Number as Figure 6.

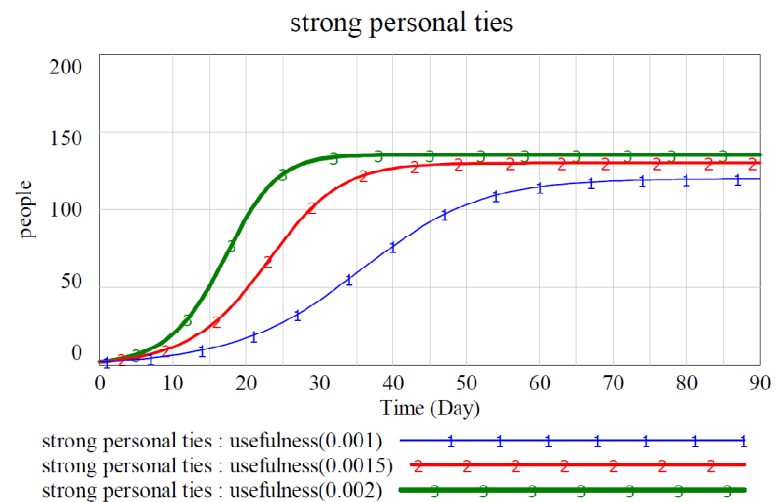

Fig. 7. The Changes in the Number of Strong Personal Ties (Open SNS)

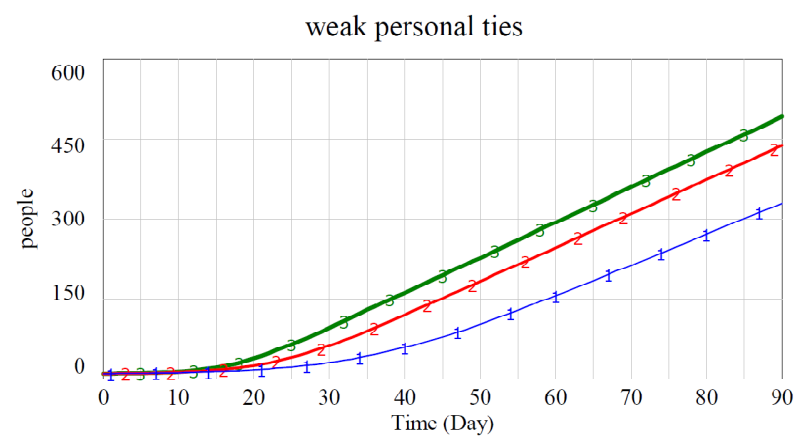

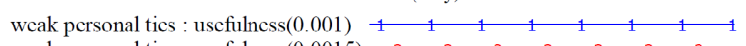
weak personal ties : usefulness $(0.0015)$ weak personal ties : usefulness $(0.002)$

Fig. 8. The Changes in the Weak Personal Ties (Open SNS)

\subsection{The Result of Simulation of Open SNS}

The simulation on Open SNS includes the structure of Close SNS, and shows the dynamic structure of weak ties. The author tried to alter the usefulness value in order to examine whether Dunbar Number applies to Open SNS as in Closed SNS as Figure 7. The result shows that the number of weak personal ties increases exponentially as Figure 8. The number of weak personal ties is affected by the number of strong personal ties, self produced contents, and collective intelligence in feedback. Different from the number of strong personal ties, the number of weak personal ties does not necessarily require a certain level of recognition, so that it is irrelevant to Dunbar Number and shows an exponentially increasing pattern.

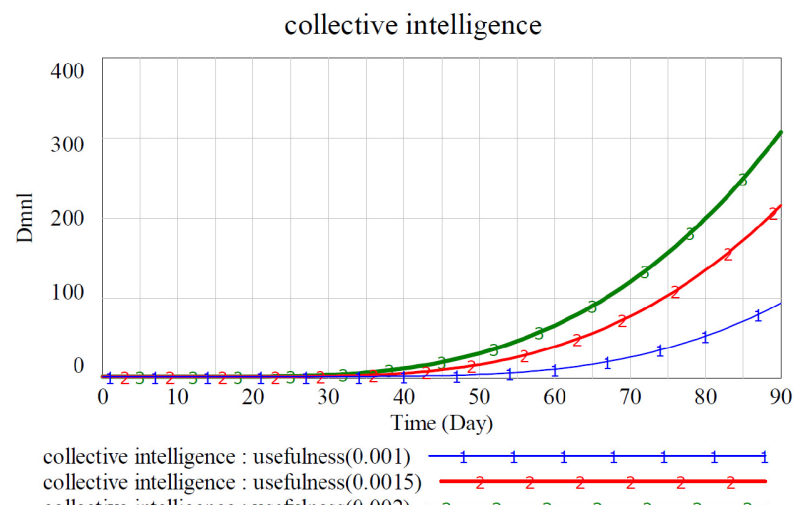

collective intelligence : usefulness $(0.0015)$

collective intelligence : usefulness $(0.002)$

Fig. 9. The Changes in Collective Intelligence Resulting from the Strengthening of Strong Ties (Open SNS)

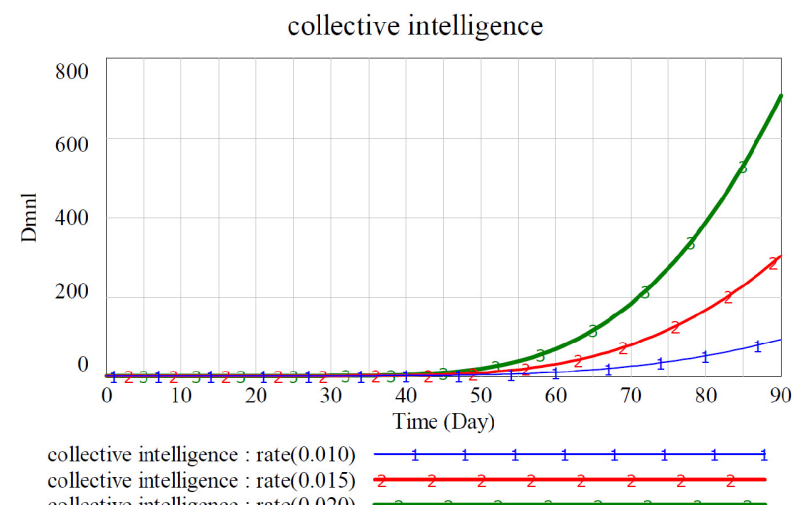

collective intelligence : rate $(0.015)$
collective intelligence : rate $(0.020)$

Fig. 10. The Changes in Collective Intelligence Resulting from the Strengthening of Weak Ties (Open SNS)

The changes in usefulness value that has a direct impact on the strengthening of strong ties in Open SNS are insignificant in the structure of BOT as Figure 9. The only difference is the slope, in other words, the degree to which it is strengthened more exponentially. It may be more appropriate to interpret that the strengthening of strong ties leads to the increase in the number of weak personal ties rather than the expansion of collective intelligence due to the contents created by the personal ties with such strong link, and thus the weak ties increases the collective intelligence through the production and reliability enhancement of contents. As shown above, the strengthening of strong ties is limited to the value of Dunbar Number and the scale of contents and collective intelligence 
generated through such strong ties was not significantly high.

The result reveals that an attempt to strengthen strong ties is meaningless due to the constraint of Dunbar Number in a system only with strong ties, whereas in a system where weak ties coexist, an attempt to strengthen strong ties is significant in a way that manifests collective intelligence. The strengthening of weak ties in Open SNS leads to the increase in contents and it consequently manifests collective intelligence as Figure 10. The increase in contents results in the increase in wrong information and thus deteriorates the quality of contents, but raises the reliability, so that it contributes to the overall increase in collective intelligence.

\section{CONCLUSION}

The study reveals the following results and implications. First, Dunbar Number applies to strong ties both in Closed and Open SNS. The results are equivalent to the conclusion obtained from the application of the law by Robin Dunbar to the 'friend tie building' in an online community through SNS.

Second, the presence of weak ties within a system leads to the emergence of collective intelligence through weak ties even in case where Dunbar Number applies, so that an attempt to strengthen weak ties is required. In case of closed system only with strong ties, its collective intelligence tends to converge into a certain level, however strong ties are strengthened. In contrast, the strengthening of strong ties lead to the strengthening of weak ties in case of a system where strong and weak ties coexist, so that the strengthening of strong ties have an indirect impact on weak ties as well as are a significantly attempt to make collective intelligence emerge.

Third, the study confirms the fact that the core in the emergence of collective intelligence is on weak ties rather than strong ties. It was shown that the impact of strengthening strong ties on the increase of collective intelligence is less influential than that of weak ties. The result supports 'The strength of Weak Ties' proposed by Mark Granovetter [6].

The study has a scholarly implication that it attempted to adopt the perspective of structuralism, which argues that 'structure results in behavior', beyond the perspective of behavioralism, which explains that 'behavior results in behavior' in developing the theoretical system for SNS. In a study in complex system such as SNS and collective intelligence, inductive logic-based behavioralism relying on empirical data is impossible to obtain basic materials, and it is also difficult to suggest a policy alternative to ideally change the future behaviors only with apparent behaviors [9]. However, the System Dynamics adopted in this study is an effective means to overcome the limitation and problem of behavioralism by utilizing a holon perspective that accepts both structuralism and behavioralism.

In addition, the study has a policy implication that it proposes the way in which SNS is designed and operated in order to make collective intelligence emerge. It is certainly important to create a system or policy that contributes to overcome the model of Dunbar Number, but it is not necessary to restrict the expansion of network merely in order to comply with Dunbar Number.
For collective intelligence to emerge in an effective manner, more people are required to share more diverse ideas and opinions. In short, Dunbar Number applies only to strong ties that require a certain level of recognition, and thus in case of weak ties, our network is likely to be expanded even to infinity, and in such small world of network, a genuine collective intelligence is expected to emerge.

Notwithstanding, the current study has limitations as described below and they need to be considered in future research. The major limitation is originated from the fact that the value of a variable that is set by applying NUMBER(Normalized Unit Modeling By Elementary Relationship). NUMBER is very useful in that you can compare the relative behavior between variables to examine the effects of major policy. However NUMBER is not enough to explain a variety of real-world phenomena. Thus, future studies should consider the use of empirical data and sophisticated models designed.

\section{REFERENCES}

[1] C. Mackay, Extraordinary Popular Delusions and the Madness of Crowds, Harmony Books, New York, 1980.

[2] G. Le Bon, Psychologie des foules, F. Alcan, Paris, 1898

[3] J. Surowiecki, The Wisdom of Crowds, Doubleday Anchor, New York, 2004.

[4] K. V. Frisch, The Dance Language and Orientation of Bees, Leigh E. Chadwick, trans. Harvard University Press, Cambridge, 1993.

[5] D. H. Meadows, The Unavoidable A Priori : Elements of the System Dynamics Method, The MIT Press, Massachusetts, 1980.

[6] M. S. Granovetter, "The strength of Weak Ties", American Journal of Sociology, vol. 78, no. 6, pp. 13601380, 1973.

[7] P. Levy, Collective Intelligence, Basic Books, Arizona, 1997.

[8] R. Dunbar, Grooming, Gossip, and the Evolution of Language, Harvard University Press, Cambridge, 1998.

[9] S. W. Kim, "Reinterpretation of the Learning Organization from the Oriental Perspectives," Korean System Dynamics Review, vol. 9, no. 1, pp. 33-56, 2008.

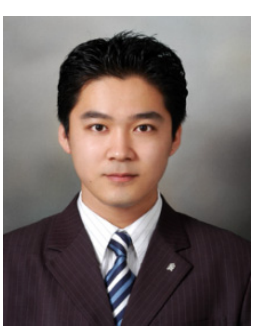

Tae- Won Kim

$\mathrm{He}$ received the M.S. in Management Information Systems from Chungbuk National University, Korea in 2008. Since th en, He Completed the course of a doctorate in Management Information Systems from Chungbuk national university, Korea in 2010. His current research interests focus on Social Network Services, Knowledge Management, e-Government and Green IT. 


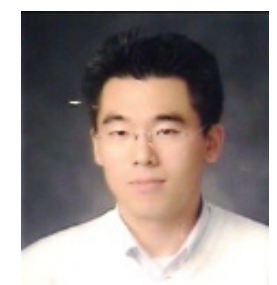

\section{Jae-Rime Jung}

$\mathrm{He}$ received the M.S. in Management Information Systems from Chungbuk National University, Korea in 2010. He is a Ph.D. Candidate of Management Information Systems at Chungbuk National University. He research interests include Knowledge Mana gement, Service Science, Theory of Constraints and System Dynamics.

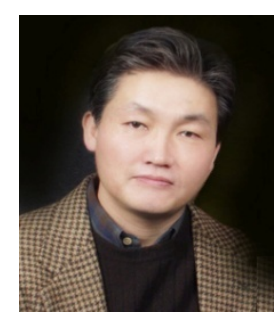

\section{Sang-Wook Kim}

$\mathrm{He}$ is a professor of MIS at Chungbuk National University, Korea. He received a Ph.D. in Management Information Systems from University of NebraskaLincoln, USA in 1987. His major books are Information Resource Management, Information Management, System Thinking and Scenario Planning. His main areas of interest include Knowledge Management, Information Strategy, eGovernment, System Dynamics among others. 